\title{
Das asymptomatische Stadium der Mitralendokardiose - Neue Erkenntnisse zur Therapie
}

\author{
Michael Deinert
}

Bisher gab es keine nachweislich wirksame Therapie im asymptomatischen Stadium der Mitralendokardiose des Hundes. Daher existierte kein therapeutischer Konsens für dieses meist 3-5 Jahre dauernde Stadium. In der EPIC-Studie (Effect of Pimobendan in Dogs with Preclinical Myxomatous Mitral Valve Disease and Cardiomegaly) konnte bei dieser häufigen Herzkrankheit nun erstmals die Wirksamkeit einer medikamentösen Therapie für das asymptomatische Stadium mit Kardiomegalie nachgewiesen werden.

\section{Einleitung}

Die Mitralendokardiose (myxomatösdegenerative AV-Klappeninsuffizienz) ist die häufigste Herzkrankheit des Hundes. $\mathrm{Ab}$ dem 13. Lebensjahr sind etwa $85 \%$ aller Hunde davon betroffen und in $75 \%$ aller Fälle von kongestivem Herzversagen ist die Ursache eine Mitralendokardiose.

Eine hinreichende Verdachtsdiagnose ist aufgrund des charakteristischen Herzgeräuschs bereits auskultatorisch, also mit einfachen diagnostischen Mitteln möglich. In der Praxis führt dies nahezu täglich zu der Fragestellung, wie mit dem Auskultationsbefund Mitralgeräusch weiter verfahren werden sollte.

Das weitere Vorgehen konzentriert sich zunächst auf ein korrektes „Staging“, also eine Zuordnung der Krankheit in einen Schweregrad. Dieser Artikel stellt die Kriterien für das Staging vor und vergleicht diese mit den Einschlusskriterien der Therapiestudien. Aktueller Anlass ist die Publikation der Ergebnisse der sog. EPIC-Studie zur Therapie der Mitralendokardiose im asymptomatischen Stadium mit Kardiomegalie, die ausführlicher vorgestellt werden soll.

\section{Signalement und klinische Untersuchung}

Das Signalement von Patienten, bei denen diese Krankheit am wahrscheinlichsten vorkommt, lautet wie folgt:

- Vertreter der kleinen Rassen, Toy- und Zwergrassen, insbesondere Yorkshire Terrier, Jack Russell Terrier, Dackel, Pudel, Chihuahua und weitere.

- Männliche Tiere sind häufiger betroffen als weibliche.

- Bei der Erstdiagnose eines Herzgeräuschs sind die Hunde i.d.R. älter als 7 Jahre.

Eine Ausnahme stellt der Cavalier King Charles Spaniel dar, bei dem je nach untersuchter Population bereits im Alter von 5 Jahren bei etwa 50\% der Hunde ein Herzgeräusch festgestellt werden kann. Je jünger die Patienten bei der Erstdiagnose sind, umso wahrscheinlicher erreichen sie eines Tages das Stadium des kongestiven Herzversagens.

\section{Herzgeräusch}

Eine Mitralinsuffizienz ist durch ein holosystolisches, bandförmiges oder Decrescendo-Geräusch charakterisiert. Das Punctum maximum liegt im Bereich der linken Herzspitze. Ein gleichlautes Geräusch auf der rechten Seite weist auf eine gleichzeitig vorliegende Trikuspidalinsuffizienz hin. Die Lautstärke kann über alle Grade reichen (von I-VI/VI) und korreliert grob mit dem Schweregrad [7] ( $\triangleright$ Tab. 1). Musikalische (quietschende, Möwenschrei-ähnliche) Geräusche deuten ebenso wie Lautstärkegrade I-II/VI auf eine geringgradige Regurgitation hin. Die Lautstärkegrade V und VI wiederum sprechen für eine hochgradige Regurgitation. Eine größere Unsicherheit besteht dagegen bei den Lautstärkegraden III und IV, die sowohl im asymptomatischen Stadium mit und ohne Kardiomegalie wie auch im symptomatischen Stadium auftreten können.

\section{Lungenödem}

Das symptomatische Stadium der Mitralendokardiose ist durch ein Lungenödem gekennzeichnet. Das früheste und sensitivste (aber nicht spezifische) klinische Symptom dafür ist eine erhöhte Ruheatemfrequenz. Durch die Polypnoe wird der erschwerte Gaswechsel durch die angestaute Ödemflüssigkeit in der kapillär-alveolären Diffusionsstrecke kompensiert. Dieses Symptom tritt früher auf als radiologische Veränderungen, die sich dann als interstitielles oder alveoläres Lungenödem zeigen.

\section{konkret}

Ab einer Ruheatemfrequenz $>35 / \mathrm{min}$ besteht der Verdacht auf ein Lungenödem und der Besitzer sollte seinen Hund vorstellen.

Tab. 1 Übersicht über die Einteilung der Lautstärkegrade eines Herzgeräuschs.

\begin{tabular}{|c|c|}
\hline $\begin{array}{l}\text { Lautstärke- } \\
\text { grad (I-VI) }\end{array}$ & Beschreibung \\
\hline I & $\begin{array}{l}\text { das leiseste, gerade noch } \\
\text { hörbare Geräusch, meist } \\
\text { erst nach einiger Zeit in } \\
\text { einem leisen Raum hörbar }\end{array}$ \\
\hline II & leise, aber sofort hörbar \\
\hline III & mittellaut \\
\hline IV & laut \\
\hline V & $\begin{array}{l}\text { sehr laut mit palpierbarem } \\
\text { Brustwandschwirren }\end{array}$ \\
\hline VI & $\begin{array}{l}\text { bereits hörbar, ohne das } \\
\text { Stethoskop vollständig auf } \\
\text { die Brustwand aufzusetzen }\end{array}$ \\
\hline
\end{tabular}


Bei einem manifesten, alveolären Lungenödem kann die Atemfrequenz auf Werte bis zu 100/min ansteigen.

\section{Husten}

Das Symptom Husten ohne gleichzeitig vorliegende Polypnoe sollte nicht als Anzeichen für kongestives Herzversagen interpretiert werden. Beim genannten Signalement (kleiner, alter Hund) besteht statistisch gesehen Multimorbidität. Hunde im Alter von 10 Jahren leiden durchschnittlich an 3-4 Krankheiten und häufig ist eine Tracheobronchomalazie oder eine chronische Bronchitis dabei. Als Hinweis auf eine Tracheobronchomalazie können durch Freude oder Aufregung triggerbare, fremdkörperartige Hustenanfälle angesehen werden, bei denen andererseits aber auch längere, beschwerdefreie Intervalle bestehen. Die Hunde zeigen dann keine Leistungsschwäche und keine Polypnoe. Eine gleichzeitig vorliegende Kardiomegalie kann diese Hustenanfälle begünstigen.

Der Hustentyp, der tatsächlich durch ein Lungenödem ausgelöst wird, ist eher ein spätes Symptom, das durch die hochgeflimmerte Flüssigkeit zu den Hustenrezeptoren der großen Bronchien ausgelöst wird. In diesem Fall liegen aber bereits schwere und permanente klinische Symptome durch ein höhergradiges alveoläres Lungenödem mit entsprechenden radiologischen Veränderungen vor. Je nach individueller Progredienz hat sich dies schon Tage zuvor durch eine erhöhte Ruheatemfrequenz angekündigt.
Dies verdeutlicht die Bedeutung der Messung der häuslichen Ruheatemfrequenz bei bekannter asyptomatischer Mitralendokardiose als Frühzeichen des Übergangs ins kongestive Herzversagen.

\section{Weitere Symptome}

Andere Symptome wie Leistungsschwäche, Hecheln, Müdigkeit, Appetitlosigkeit oder Gewichtsverlust sind nicht spezifisch für eine Herzkrankheit und können mit gleicher Wahrscheinlichkeit orthopädische, endokrinologische oder gastrointestinale Ursachen haben. In den Guidelines von 2009 [1] wird aus diesem Grund in allen Fällen, in denen die klinischen Symptome nicht eindeutig einem kongestiven Herzversagen mit dem radiologischen Befund eines Lungenödems zuzuordnen sind, eine echokardiografische Untersuchung und gegebenenfalls eine weitere Diagnostik empfohlen.

\section{Stadien einer Mitralendokardiose}

Die für das Tier modifizierte NYHA-Klassifikation wird kaum noch angewendet und wurde durch verfeinerte Klassifikationssysteme ersetzt. Im ACVIM-Klassifikationsschema gibt es zunächst ein Stadium A für Tiere, die aufgrund der Rasse oder Familienanamnese ein erhöhtes Risiko für eine Herzkrankheit besitzen. In Stadium B ist eine Mitralinsuffizienz nachweisbar, aber das Stadium des kongestiven Herzversagens wurde noch nicht erreicht. Dieses asymptomatische Stadium wird weiter unterteilt in B1

\section{Abkürzungen \\ ACVIM: American College of Veterinary Internal Medicine \\ CHIEF: Canine Heart Failure Internatio- nal Expert Forum \\ NYHA: New York Heart Association \\ RAAS: Renin-Angiotensin-Aldosteron- System}

ohne Kardiomegalie und B2 mit Kardiomegalie. In Stadium C zeigt der Patient Anzeichen eines kongestiven Herzversagens und in Stadium D liegt ein kongestives Herzversagen in einer schweren Form vor, die refraktär auf (Standard-) Therapie ist. Die Stadien C und D können noch in ambulante und stationäre Therapie unterteilt werden. Nach Erreichen des Stadiums C ist auch unter erfolgreicher Therapie keine Rückkehr in Stadium B mehr möglich.

Die ähnliche CHIEF-Klassifikation kennt daher sinnvollerweise noch ein Stadium C1. Im Stadium C1 befinden sich Patienten, die nach Erreichen des Herzversagens medikamentös stabilisiert wurden und dadurch wieder asymptomatisch sind. Die CHIEF-Klassifikation unterscheidet im Stadium B jedoch nicht zwischen Patienten ohne oder mit Kardiomegalie. In $>$ Tab. 2 werden deshalb die ACVIM- und CHIEF-Klassifikationen kombiniert dargestellt. Abb. 1 zeigt eine Übersicht über die radiologischen Befunde und $>$ Abb. 2 über den Verlauf der Stadien.

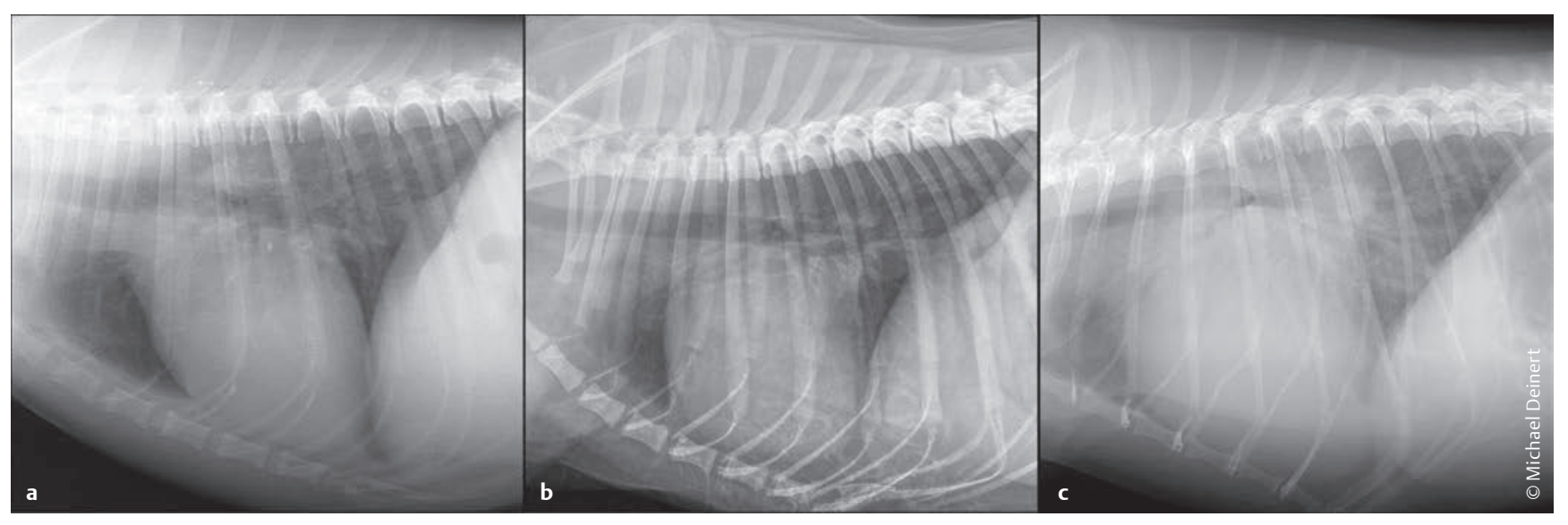

Abb. 13 Thoraxröntgenaufnahmen von Hunden in verschiedenen Stadien einer Mitralendokardiose: a Stadium B1 ohne Kardiomegalie (VHS 9,5) und ohne erkennbare linksatriale Vergrößerung; b Stadium B2 mit Kardiomegalie (VHS 11,3) mit linksatrialer Vergrößerung (Ausziehung am kaudodorsalen Herzrand); c Stadium C im kongestivem Herzversagen, charakterisiert durch ein alveoläres Lungenödem zentral im Hauptlappen. 


\section{Stadium A}

$\mathrm{Zu}$ diesem Stadium gehören alle Hunde, deren Signalement ein erhöhtes Risiko für eine Mitralendokardiose vermuten lässt oder wenn deren Geschwister- oder Elterntiere bekanntermaßen erkrankt sind. Dazu zählt insbesondere der Cavalier King Charles Spaniel. Die Empfehlung lautet: Monitoring durch jährliche Auskultation und eine erste Beratung des Besitzers über Anzeichen der Krankheit und die Bedeutung der Messung der Ruheatemfrequenz.

\section{Asymptomatische Stadien B1 und B2}

In Stadium B ist ein Mitralgeräusch auskultierbar, nicht selten als Zufallsbefund im Rahmen einer Routineuntersuchung. Das bedeutet im Umkehrschluss, dass dem Besitzer bislang keine Symptome aufgefallen sind. Die Patienten befinden sich dann im asymptomatischen Stadium einer Mitralendokardiose.

Etwaige vorhandene Symptome sollten kritisch und sorgfältig auf eine kardiale Genese hin untersucht werden. Dazu ist in den meisten Fällen eine Thoraxröntgenstudie, häufig auch eine Echokardio- grafie notwendig. Anhand dieser bildgebenden Verfahren kann die Herzgröße vermessen werden und dadurch eine $\mathrm{Zu}$ ordnung in B1 oder B2 erfolgen.

\section{Asymptomatisches Stadium B1 ohne Kardiomegalie}

Patienten in diesem Stadium haben mit großer Wahrscheinlichkeit nur ein leises bis mittellautes Herzgeräusch (Grad IIII/VI). Das Vorliegen einer respiratorischen Arrhythmie oder von Herzfrequenzen $<130 /$ min sprechen gegen eine Dekompensation. In diesem Stadium ist per Definition noch keine Herzvergröße-

Tab. 2 Übersicht, Befunde und Therapieempfehlungen basierend auf einer Kombination aus dem CHIEF- und ACVIM-Klassifikationssystem für die verschiedenen Schweregrade einer Mitralendokardiose.

\begin{tabular}{|c|c|c|c|c|c|c|}
\hline $\begin{array}{l}\text { Stadium/Zustand } \\
\text { des Patienten }\end{array}$ & $\begin{array}{l}\text { ACVIM/ } \\
\text { CHIEF- } \\
\text { Staging }\end{array}$ & Befunde & Studie & $\begin{array}{l}\text { Ziel der } \\
\text { Therapie }\end{array}$ & Wirksamkeit & Empfehlung \\
\hline $\begin{array}{l}\text { keine Herzkrank- } \\
\text { heit nachweisbar, } \\
\text { aber Risikopatient } \\
\text { für eine Mitral- } \\
\text { endokardiose, } \\
\text { z. B. wegen Rasse, } \\
\text { Familien- } \\
\text { anamnese etc. }\end{array}$ & A & keine & keine & keine Therapie & - & $\begin{array}{l}\text { jährliche Auskultation, } \\
\text { Besitzer über die Bedeutung/ } \\
\text { Messung der Ruheatem- } \\
\text { frequenz informieren }\end{array}$ \\
\hline $\begin{array}{l}\text { asymptomatisch } \\
\text { ohne Kardio- } \\
\text { megalie }\end{array}$ & B1 & $\begin{array}{l}\text { Mitralgeräusch } \\
\text { (meist I-III/VI), } \\
\text { keine Kardio- } \\
\text { megalie: Herz- } \\
\text { größe im Echo } \\
\text { und Röntgen im } \\
\text { Referenzbereich }\end{array}$ & SVEP & $\begin{array}{l}\text { Verlängerung } \\
\text { der Zeit bis } \\
\text { zum Herz- } \\
\text { versagen } \\
\text { (Lungenödem) }\end{array}$ & nein & keine Therapie \\
\hline $\begin{array}{l}\text { asymptomatisch } \\
\text { mit Kardiomegalie }\end{array}$ & B2 & $\begin{array}{l}\text { Mitralgeräusch } \\
\text { (meist III-V/VI); } \\
\text { Kardiomegalie: } \\
\text { Herzgröße im } \\
\text { Echo und Röntgen } \\
\text { vergrößert, aber } \\
\text { kein Lungenödem }\end{array}$ & $\begin{array}{l}\text { SVEP, } \\
\text { Vetproof, } \\
\text { EPIC }\end{array}$ & $\begin{array}{l}\text { Verlängerung } \\
\text { der Zeit bis } \\
\text { zum Herz- } \\
\text { versagen } \\
\text { (Lungenödem) }\end{array}$ & $\begin{array}{l}\text { ja, für } \\
\text { Pimobendan }\end{array}$ & $\begin{array}{l}\text { Pimobendan in empfohlener } \\
\text { Standarddosis }\end{array}$ \\
\hline $\begin{array}{l}\text { stabilisiert mit } \\
\text { medikamentöser } \\
\text { Therapie } \\
\text { (Lungenödem war } \\
\text { nachgewiesen } \\
\text { und wird } \\
\text { erfolgreich } \\
\text { therapiert) }\end{array}$ & C1 & $\begin{array}{l}\text { Mitralgeräusch } \\
\text { (meist III-VI/VI), } \\
\text { keine respiratori- } \\
\text { sche Arrhythmie } \\
\text { mehr vorhanden }\end{array}$ & $\begin{array}{l}\text { QUEST, } \\
\text { IMPROVE, } \\
\text { Bernay }\end{array}$ & $\begin{array}{l}\text { Rezidiv des } \\
\text { Lungenödems } \\
\text { verhindern, } \\
\text { gute Lebens- } \\
\text { qualität, } \\
\text { Gewinn } \\
\text { Lebenszeit }\end{array}$ & $\begin{array}{l}\text { für alle rechts } \\
\text { genannten } \\
\text { Medikamente }\end{array}$ & $\begin{array}{l}\text { dauerhafte Therapie p.o.: } \\
\text { 1. Diuretikum (Furosemid/ } \\
\text { Torasemid) } \\
\text { 2. Pimobendan } \\
\text { 3. RAAS-Blockade } \\
\text { (ACE-Hemmer-Spironolac- } \\
\text { ton-Kombination): } \\
\text { sog. „Tripeltherapie mit } \\
4 \text { Wirkstoffen“ }\end{array}$ \\
\hline $\begin{array}{l}\text { symptomatisch, } \\
\text { da dekompensiert } \\
\text { (Lungenödem } \\
\text { vorhanden) }\end{array}$ & $C 2-D$ & $\begin{array}{l}\text { Mitralgeräusch } \\
\text { (meist III-VI/VI), } \\
\text { Lungenödem, } \\
\text { ggf. Aszites, } \\
\text { pulmonale } \\
\text { Hypertension, } \\
\text { Arrhythmien, Syn- } \\
\text { kopen }\end{array}$ & - & $\begin{array}{l}\text { ins Stadium C1 } \\
\text { zu kommen } \\
\text { (Beseitigung } \\
\text { der Konges- } \\
\text { tionszeichen) }\end{array}$ & $\begin{array}{l}\text { in C2 gutes } \\
\text { Ansprechen, } \\
\text { in } D \text { indivi- } \\
\text { duell, je nach } \\
\text { Schweregrad } \\
\text { und Kompli- } \\
\text { kationen } \\
\text { (refraktär) }\end{array}$ & $\begin{array}{l}\text { Furosemid i.v./i.m., } \mathrm{O}_{2} \text {; in C2, } \\
\text { dann wie oben, in D meist } \\
\text { weitere Maßnahmen } \\
\text { (zusätzliches Diuretikum, } \\
\text { Nachlastsenker, Lungen- } \\
\text { drucksenkung, Aszites- } \\
\text { punktion), Antiarrhythmika, } \\
\text { Intensivmaßnahmen }\end{array}$ \\
\hline
\end{tabular}


rung nachweisbar. Zum Nachweis sollte dem Besitzer eine Thoraxröntgenstudie empfohlen werden.

Die Herzgröße kann anhand der Herzwirbelsumme (VHS, vertebral heart score) vermessen werden. Als obere Grenze werden generell 10,5 Wirbel angesehen. Jedoch zeigen zahlreiche Rassen physiologischerweise höhere Werte. Insbesondere bei brachyzephalen Rassen kann ein VHS > 12 noch physiologisch

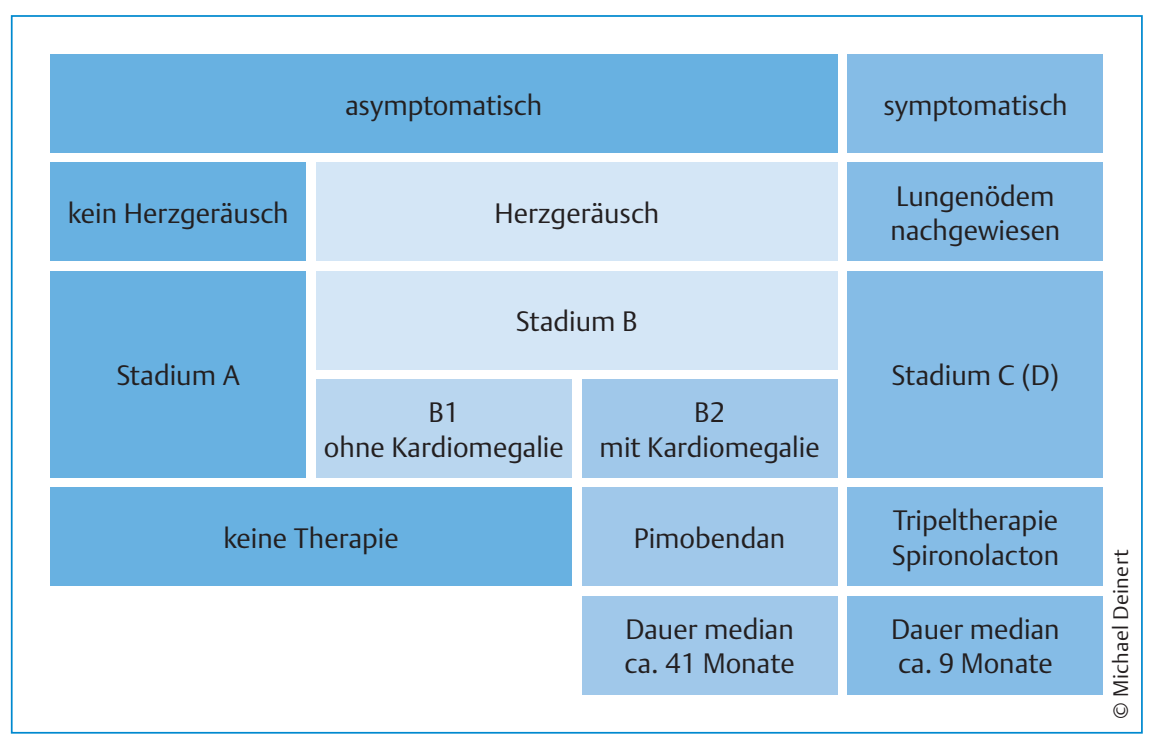

Abb. 2 Schematischer Verlauf der Stadien einer Mitralendokardiose. Weiteres dazu im Text.
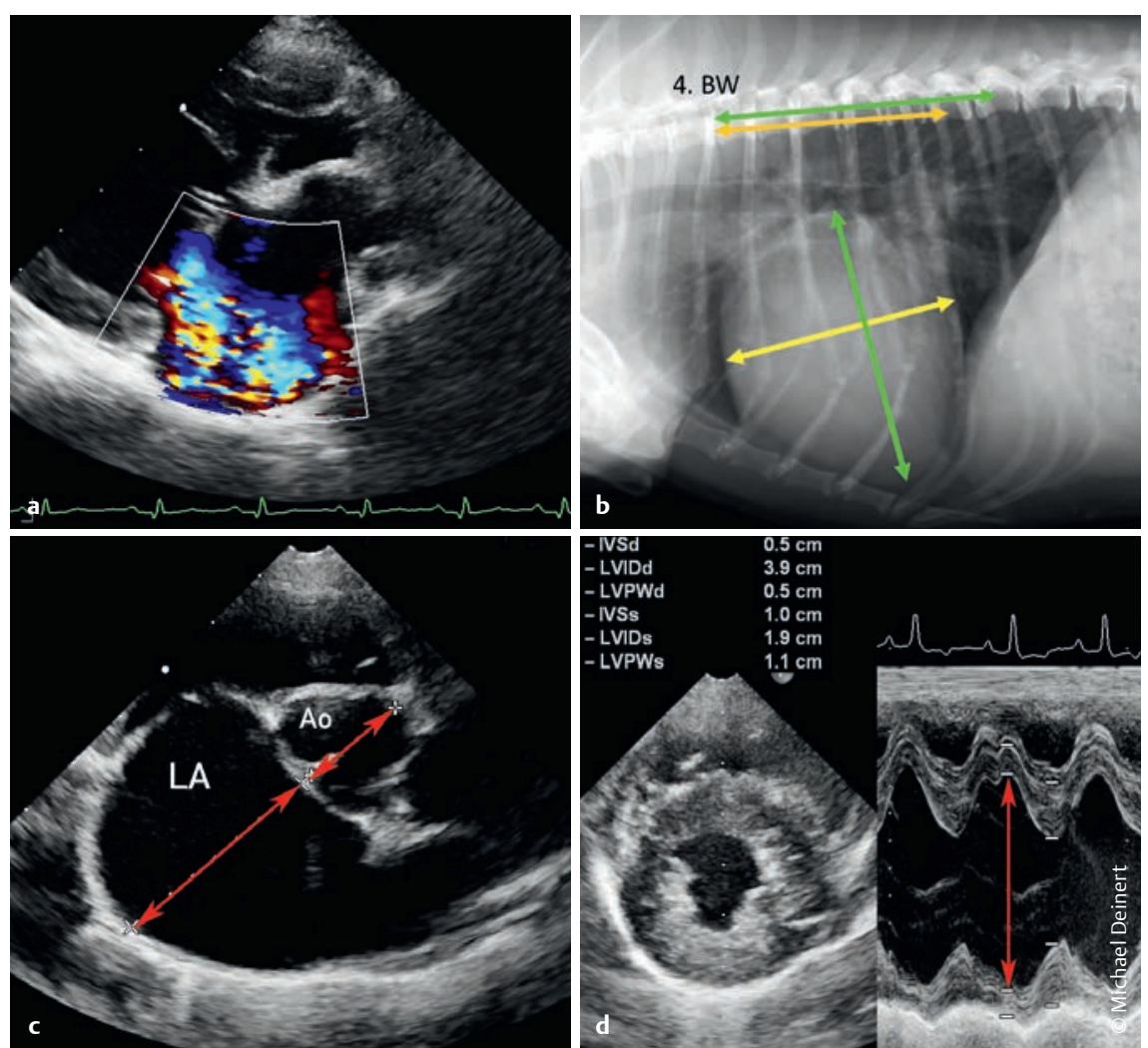

Abb. 3 Übersicht über die Nachweismethoden der wichtigsten Einschlusskriterien für die EPIC-Studie. a Echokardiografischer Nachweis von Klappenveränderungen und einer Mitralinsuffizienz. b Bestimmung der Herzwirbelsumme im Röntgenbild. c Bestimmung des Quotienten linkes Atrium zur Aorta in der kurzen Achse. d Messung des linksventrikulären diastolischen Durchmessers LVDd zur Errechnung des gewichtsnormierten Wertes LVDdN (Formel: LVDdN = LVDd (cm)/Körpergewicht $(\mathrm{kg})^{0,294}$ [5].

sein. Unter anderem können dafür auch fusionierte oder Keilwirbel in der Brustwirbelsäule verantwortlich sein [8].

Bei einer erhöhten Herzwirbelsumme $>$ 10,5 wird zur Absicherung eine echokardiografische Untersuchung empfohlen.

In diesem Stadium wird aufgrund der Ergebnisse der SVEP-Studie keine medikamentöse Therapie empfohlen $[1,9]$.

\section{Asymptomatisches Stadium B2 mit Kardiomegalie}

Im Stadium B2 ist eine Herzvergrößerung nachweisbar, jedoch bestehen keine Anzeichen für ein kongestives Herzversagen. Ein trockener, anfallsweiser Husten spricht für eine Kombination aus Bronchomalazie und Kardiomegalie und sollte nicht als kongestives Herzversagen interpretiert werden. Dazu ist aus klinischer Sicht die Erfassung der Ruheatemfrequenz besser geeignet.

Der Nachweis einer Kardiomegalie kann röntgenologisch erfolgen. Gleichzeitig können dabei weitere wichtige thorakale Strukturen, insbesondere die Lungenvenen und das Lungenparenchym beurteilt werden. In der EPIC-Studie lag der VHS der Patienten in diesem Stadium zwischen 10,9 und 12. Die unter Stadium B1 genannten Limitierungen der VHSMessung sollten jedoch beachtet werden. Daher ist gerade im Stadium B2 eine echokardiografische Untersuchung unbedingt zu empfehlen, um die Einschlusskriterien der EPIC-Studie und damit die Therapieindikation mit Pimobendan für den einzelnen Patienten sicherzustellen [4] ( Abb. 3).

\section{Stadien C und D}

Patienten in diesen Stadien haben ein kongestives Herzversagen. Typische klinische Anzeichen sind

- erhöhte Atemfrequenz,

- Tachykardie und

- lautes Mitralgeräusch ( $\geq$ IV/VI).

Der Nachweis erfolgt durch entsprechende Veränderungen im Thoraxröntgen: Diese sind ein interstitielles oder alveoläres Verschattungsmuster zentral in den Hauptlappen der Lunge, dabei ist der rechte Hauptlappen stärker betroffen als 
der linke. Gleichzeitig sind vergrößerte Lungenvenen und eine Kardiomegalie mit linksatrialer Vergrößerung zu erwarten. In der QUEST-Studie wurde die Wirksamkeit von Pimobendan in diesem Stadium nachgewiesen. Der VHS der Patienten in dieser Studie lag zwischen 11,5 und 13,5 [6].

Die medikamentöse Dauertherapie besteht aus Diuretika, Pimobendan und RAAS-Blockade (Tripeltherapie, $>$ Tab.3). Als Diuretika sind Furosemid oder Torasemid verfügbar. Zur RAASBlockade werden ACE-Hemmer eingesetzt. Bei etwa 30-50\% der Patienten kann es unter dieser Therapie zu einem sog. Aldosteron-Escape kommen. Deshalb und aufgrund der Ergebnisse einer Therapiestudie kann die zusätzliche Gabe von Spironolacton zur sequenziellen RAAS-Blockade in diesem Stadium sinnvoll sein [3]. Dazu eignet sich das Kombinationspräparat aus Benazepril und Spironolacton (Cardalis ${ }^{\circledR}$, Fa. Ceva). Die medikamentöse Therapie kann in diesem Stadium nicht mehr abgesetzt werden.

Diätetische Maßnahmen beinhalten eine adäquate kalorische Versorgung, um einer kardialen Kachexie entgegenzuwirken, sowie ein leicht reduzierter Natriumgehalt.

\section{Therapiestudien}

Für eine Therapie können abhängig vom Stadium der Krankheit verschiedene Ziele formuliert werden. Im symptomatischen Stadium sollen Symptome des kongestiven Herzversagens beseitigt und dem Patienten anschließend eine möglichst lange Phase mit guter Lebensqualität ermöglicht werden. Der therapeutische Erfolg wird durch Symptomfreiheit, Lebensqualität und Zeit definiert.

Im asymptomatischen Stadium ist das Ziel dagegen nicht die Beseitigung von Symptomen, sondern eine möglichst lange Konservierung des Status quo und ein späteres Eintreten ins symptomatische Stadium. Da die Wirksamkeit einer Therapie in diesem Stadium alleine durch Zeit definiert ist (in Studien „time to event“ oder „endpoint“), kann der Nachweis nur in gut angelegten und über eine ausreichend lange Zeitdauer laufenden Studien erfolgen.

Kennzeichen guter Therapiestudien sind prospektive Planung, Vergleich mit einer Kontrollgruppe strukturgleicher Probanden, (Doppel-)Verblindung sowie Randomisierung bei der Gruppenzuteilung (prospective, randomized, controlled, blinded trial, PRCBT). Weiterhin sollten Ein- und Ausschlusskriterien sowie Studienendpunkte eindeutig und objektiv definiert sein. Diese dürfen insbesondere in Multicenterstudien wenig Spielraum für eine subjektive Einschätzung bieten.

Die im asymptomatischen Stadium der Mitralendokardiose durchgeführten Studien (VETPROOF-, EPIC-, SVEP-Studie [2, 4, 9]) erfüllen diese Anforderungen. In den bisherigen Studien für das asymptomatische Stadium der Mitralendokardiose ergab sich keine Wirksamkeit für eine Therapie mit ACE-Hemmern $[2,9]$. Der Studienendpunkt „kongestives Herzversagen“ wurde 
Tab. 3 Übersicht über die wichtigsten Herzmedikamente zur oralen Dauertherapie im Stadium C (und D) einer Mitralendokardiose. Beipackzettel und detaillierte Fachinformationen sollten beachtet werden.

\begin{tabular}{|c|c|c|c|c|}
\hline $\begin{array}{l}\text { Wirkstoff- } \\
\text { gruppe }\end{array}$ & Wirkstoff & $\begin{array}{l}\text { Handelsnamen } \\
\text { (Beispiele) }\end{array}$ & Dosierung & Bemerkungen \\
\hline \multicolumn{5}{|c|}{ Inodilatator (Phosphodiesterasehemmer) } \\
\hline- & Pimobendan & Vetmedin $^{\circledR}$, Cardisure ${ }^{\circledR}$ & $0,5(0,2-0,6) \mathrm{mg} / \mathrm{kg}$, auf $2 \times \mathrm{tgl}$. p.o. & - \\
\hline \multicolumn{5}{|c|}{ Schleifendiuretika } \\
\hline \multirow[t]{2}{*}{-} & Furosemid & $\begin{array}{l}\text { Dimazon }^{\circledR} \text {, Furotab } \\
\text { Libeo }^{\circledR}\end{array}$ & $2 \mathrm{mg} / \mathrm{kg}, 1-2 \times$ tgl. p.o. & $\begin{array}{l}\text { Gabe auch bis zu } 4 \mathrm{mg} / \mathrm{kg} \text { und } \\
3 \times \mathrm{tgl} \text {. möglich; } \\
\text { Dosierungen für orale } \\
\text { Erhaltungstherapie, im Notfall } \\
\text { parenterale Gabe }\end{array}$ \\
\hline & Torasemid & Upcard $^{\circledR}$ & 0,1-0,6 mg/kg, $1 \times$ tgl. p.o. & $1 / 20$ der Furosemid-Tagesdosis \\
\hline \multicolumn{5}{|c|}{ Thiaziddiuretika } \\
\hline- & Hydrochlorothiazid & Esidrix $^{\circledR}$ & 1-2 mg/kg, $2 \times$ tgl. p.o. & humanmedizinisch \\
\hline \multicolumn{5}{|c|}{ RAAS-Blockade } \\
\hline \multirow{4}{*}{$\begin{array}{l}\text { ACE-Hemmer } \\
\text { (Endung: -pril) }\end{array}$} & Ramipril & Vasotop $^{\circledR}$ & $0,125-0,25 \mathrm{mg} / \mathrm{kg}, 1 \times$ tgl. p.o. & - \\
\hline & Imidapril & Prilium $^{\circledR}$ & 0,25 mg/kg, $1 \times$ tgl. p.o. & flüssige Formulierung \\
\hline & Benazepril & $\begin{array}{l}\text { Benakor }^{\circledR}, \text { Benefortin }^{\circledR} \text {, } \\
\text { Fortekor }^{\circledR}, \text { Nelio }^{\circledR}\end{array}$ & 0,25-0,5 mg/kg, $1 \times$ tgl. p.o. & Dosis kann verdoppelt werden \\
\hline & Enalapril & Enacard $^{\circledR}$ & 0,5 mg/kg, $1 \times$ tgl. p.o. & Dosis kann verdoppelt werden \\
\hline \multicolumn{5}{|c|}{ Aldosteron-Rezeptoren-Blocker } \\
\hline- & Spironolacton & Prilactone $^{\circledR}$ & $2 \mathrm{mg} / \mathrm{kg}, 1 \times$ tgl. p.o. & - \\
\hline \multicolumn{5}{|l|}{ Kombinationen } \\
\hline- & $\begin{array}{l}\text { Benazepril + } \\
\text { Spironolacton }\end{array}$ & Cardalis $^{\circledR}$ & $\begin{array}{l}2(-4) \mathrm{mg} / \mathrm{kg} \text { (bezogen auf Spirono- } \\
\text { lactonkomponente), } 1 \times \text { tgl. p.o. }\end{array}$ & - \\
\hline
\end{tabular}

in der Placebo-Gruppe nach der gleichen Zeit ohne statistisch signifikanten Unterschied zu der Verum-Gruppe erreicht. Bis zum Erscheinen der Ergebnisse der EPIC-Studie existierte daher keine bewiesen wirksame Therapie. Umso aussagekräftiger ist unter diesem Gesichtspunkt das eindeutige Ergebnis der EPICStudie [4], die mit einem vergleichbaren Design wie die vorangegangene VETPROOF-Studie [2] angelegt wurde.

\section{Die EPIC-Studie}

In der aktuell publizierten EPIC-Studie (EPIC: Effect of Pimobendan in Dogs with Preclinical Myxomatous Mitral Valve Disease and Cardiomegaly) wurde bei Hunden im Stadium B2 einer Mitralendokardiose die Wirksamkeit von Pimobendan mit der Hypothese untersucht, dass die Zeit bis zum Erreichen des Stadiums eines kongestiven Herzversagens durch die Therapie verlängert wird. Dabei handelt es sich um die größte pros- pektive kardiologische Therapiestudie, die bislang durchgeführt wurde (Studienaufbau \ s. Kasten S. 10).

Nach Aufnahme in die Studie (Tag 0) erfolgte die 1. Kontrolluntersuchung nach 35 Tagen und danach alle 4 Monate. Folgende Untersuchungen waren vorgeschrieben:

- klinische Untersuchung (bei jedem Besuch)

- Blutdruckmessung (Tag 0)

- Laboruntersuchung und eine Echokardiografie (an Tag 0 und 35)

- Thoraxröntgen (Tag 0 und dann immer alle 8 Monate)

Der Besitzer dokumentierte die häusliche Ruheatemfrequenz und beantwortete bei jedem Besuch einen Fragebogen zur Lebensqualität des Hundes (Belastbarkeit, Verhalten, Appetit, Atmung, Husten, Auftreten von Synkopen).
Primärer Endpunkt der Studie war entweder das Auftreten von kongestivem Herzversagen oder Tod aus kardialen Gründen (kombinierter Endpunkt). Zum Nachweis des kongestiven Herzversagens mussten Thoraxröntgenbilder vorliegen, die ein Lungenödem zeigen. Die Röntgenbilder wurden daraufhin von einer Kommission beurteilt. Erst bei mehrheitlicher Übereinstimmung galt der Endpunkt kongestives Herzversagen für diesen Patienten als erreicht.

Insgesamt wurden 354 Hunde statistisch ausgewertet. Davon erreichten 162 den primären Endpunkt, während 79 diesen zum Ende der Studie noch nicht erreicht hatten (Eventrate 45,8\%). Bei den übrigen Hunden kam es zu Protokollverletzungen, Ausscheiden auf Besitzerwunsch oder Tod aus nicht kardialen Gründen. Die mediane Zeit bis zum primären (kombinierten) Endpunkt war für die Pimobendan-Gruppe mit 1228 Tagen sig- 


\section{Zusammenfassung der EPIC-Studie}

Asymptomatisches Stadium der Mitralendokardiose mit Kardiomegalie (B2)

- Studiendesign: randomisiert, doppel-verblindet, Placebo-kontrolliert in weltweit 36 Zentren

- 360 Hunde in 2 Gruppen

- Placebo vs. Pimobendan

Einschlusskriterien:

- Mindestalter von 6 Jahren

- Körpergewicht zwischen 4,1 und $15 \mathrm{~kg}$

- Mitralgeräusch $\geq$ Grad III/VI

- charakteristische Befunde einer Mitralendokardiose in der Echokardiografie

Nachweis der Kardiomegalie durch:

- Echokardiografie

- Verhältnis des linken Atriums zur Aorta in der kurzen Achse $\geq 1,6$

- gewichtsnormierten diastolischen Kammerdurchmesser $\geq 1,7$

- Thoraxröntgen

- Herzwirbelsumme > 10,5

Alle 3 Kriterien mussten für die Aufnahme in die Studie erfüllt sein.

Ausschlusskriterien:

- vorhergehendes Lungenödem

- Vorbehandlungen mit Herzmedikamenten

- klinisch relevante Tachyarrhythmien

- pulmonale Hypertension mit einem Druckgradienten $>65 \mathrm{mmHg}$ (rechter Ventrikel zu rechtem Atrium)

- andere Herzkrankheiten als eine Mitralendokardiose

Ergebnis:

Mediane Zeit bis zum primären Endpunkt für die Pimobendan-Gruppe signifikant

länger $(p=0,0038)$ :

- Pimobendan-Gruppe: 1228 Tage

- Placebo-Gruppe: 766 Tage

nifikant länger als für die Placebo-Gruppe (766 Tage, $\mathrm{p}=0,0038$ ). Für den Endpunkt kongestives Herzversagen alleine betrachtet betrug der Unterschied 1337 zu 846 Tagen $(p=0,0018)$. Dabei konnte keine nachteilige Wirkung für eine Therapie mit Pimobendan nachgewiesen werden. Bei Betrachtung der „all-causemortality“ ergaben sich längere Überlebenszeiten für die PimobendanGruppe, bei Tod aus kardialen Gründen ergaben sich keine Unterschiede zur Placebo-Gruppe. Die Therapie mit Pimobendan kann aufgrund dieser Ergebnisse als wirksam, sicher und verträglich angesehen werden.

\section{Fazit}

In der EPIC-Studie konnte die Wirksamkeit von Pimobendan in der Standarddosierung $(0,4-0,6 \mathrm{mg} / \mathrm{kg}$ auf $2 \times$ tgl. p.o.) im asymptomatischen Stadium der Mitralendokardiose mit Kardiomegalie
(Stadium B2) nachgewiesen werden. Die Zeit bis zum Auftreten eines Lungenödems (kongestives Herzversagen) oder bis zum Tod aus kardialen Gründen wurde durch die Therapie von median 766 auf 1228 Tage signifikant verlängert $(p=0,0038)$. Dies bedeutet für den einzelnen Patienten individuell eine Verlängerung des asymptomatischen Stadiums um etwa das 1,6-Fache der Zeit, die er ohne Therapie in diesem Stadium verbracht hätte. Dieses Argument kann im Besitzergespräch angeführt werden, ohne dass absolute Zahlen genannt werden müssen. Jedoch sollte der Besitzer überzeugt werden, echokardiografisch ein korrektes Staging vornehmen zu lassen (Verifizierung der Kardiomegalie).

Eine Wirksamkeit im asymptomatischen Stadium ohne Kardiomegalie (Stadium B1) wurde in dieser Studie nicht untersucht. Ob eine Therapie mit Pimobendan in diesem früheren Stadium uner- wünschte oder negative Auswirkungen hat, ist ebenfalls nicht systematisch untersucht. Die ACVIM-Empfehlungen für das asymptomatische Stadium ohne Kardiomegalie wurden in den Guidelines von 2009 klar formuliert: keine Therapie. Daran hat sich auch nach der EPIC-Studie nichts geändert.

Online zu finden unter

http://dx.doi.org/10.1055/s-0042-123786

\section{Literatur}

1 Atkins C, Bonagura J, Ettinger $S$ et al. Guidelines for the diagnosis and treatment of canine chronic valvular heart disease. J Vet Intern Med 2009; 3 (6): 1142-1150

2 Atkins CE, Keene BW, Brown WA et al. Results of the veterinary enalapril trial to prove reduction in onset of heart failure in dogs chronically treated with enalapril alone for compensated, naturally occurring mitral valve insufficiency. J Am Vet Med Assoc 2007; 231 (7): 1061-1069

3 Bernay F, Bland JM, Haggström J et al. Efficacy of spironolactone on survival in dogs with naturally occurring mitral regurgitation caused by myxomatous mitral valve disease. J Vet Intern Med 2010; 24 (2): 331-341

4 Boswood A, Häggström J, Gordon SG et al. Effect of Pimobendan in Dogs with Preclinical Myxomatous Mitral Valve Disease and Cardiomegaly: The EPIC Study-A Randomized Clinical Trial. J Vet Intern Med 2016; 30 (6): 1765-1779

5 Cornell CC, Kittleson MD, Della Torre P et al. Allometric scaling of $\mathrm{M}$-mode cardiac measurements in normal adult dogs. J Vet Intern Med 2004; 18 (3): 311-321

6 Häggstrom J, Boswood A, O'Grady M et al. Effect of pimobendan or benazepril hydrochloride on survival times in dogs with congestive heart failure caused by naturally occurring myxomatous mitral valve disease: the QUEST study. J Vet Intern Med 2008; 22 (5): 1124-1135

7 Häggström J, Kvart C, Hansson K. Heart sounds and murmurs: changes related to severity of chronic valvular disease in the Cavalier King Charles spaniel. J Vet Intern Med 1995; 9 (2): 75-85

8 Jepsen-Grant K, Pollard RE, Johnson LR. Vertebral heart scores in eight dog breeds. Vet Radiol Ultrasound 2013; 54 (1): 3-8

9 Kvart C, Häggström J, Pedersen HD et al. Efficacy of enalapril for prevention of congestive heart failure in dogs with myxomatous valve disease and asymptomatic mitral regurgitation. J Vet Intern Med 2002; 16 (1): $80-88$

\section{Dr. Michael Deinert}

Fachtierarztpraxis Am Sandpfad

Ludwig-Wagner-Straße 31, 69169 Wiesloch

m.deinert@fachtierarztpraxis-sandpfad.de 